\title{
Underwater electro-navigation in the dark
}

\author{
Vincent Lebastard*, Frédéric Boyer*, Christine Chevallereau ${ }^{\dagger}$, and Noël Servagent ${ }^{\star}$
}

\begin{abstract}
This article proposes a solution to the problem of the navigation of underwater robots in confined unstructured environments wetted by turbid waters. The solution is based on a new sensor bio-inspired from electric fish. Exploiting the morphology of the sensor as well as taking inspiration from passive electro-location in real fish, the solution turns out to be a sensory-motor loop encoding a simple behavior relevant to exploration missions. This behavior consists in seeking conductive objects while avoiding insulating ones. The solution is illustrated on experiments. It is robust and works even in very unstructured scenes. It does not require any model and is quite cheap to implement.
\end{abstract}

\section{INTRODUCTION}

In spite of its high potential interest for applications as the exploration of deep seas or the rescue missions in catastrophic conditions, underwater navigation in confined unstructured environments wetted by turbid waters is till today a challenge for robotics. Obviously, due to the fluid opacity, vision cannot be used in these conditions while the jamming caused by the multiple interfering reflections as well as the diffraction by small floating particles considerably increase the problems posed to echolocation by sonar. Moreover, electric sense can be exploited in order to perceive some differences between objects depending on their electric properties. In particular, it would be relevant for underwater exploration to design an autonomous underwater vehicle capable of seeking conductive objects while avoiding insulating ones. This difference in electric nature could for instance instantiate the difference between dead (insulating) and alive (conductive) objects, or metallic (conductive) and other minerals (insulating)... Pursuing a bio-inspired approach in robotics, in the EU funded project Angels [1], we began to study and reproduce a sense used for navigation and communication by several families of fish whose activity is principally nocturnal and whose natural habitat is the particle-charged waters of equatorial forests: the electric sense [7]. In mormyrid fish, for instance, active "electrolocation" has a range of 1 body length and is based on the emission of an electric field by polarization of the body relative to an emitter-organ in the tail [13]. The high internal conductivity of the fish's body relative to the surrounding environment focuses the emitted field lines, obliging current

* F. Boyer and V. Lebastard are with IRCCyN-Ecole des Mines de Nantes, 4, rue Alfred Kastler B.P. 20722 - 44307 Nantes Cedex 3 France.

$\dagger$ C. Chevallereau is with IRCCyN-CNRS, Ecole Centrale de Nantes, 1 rue de la Noë, 44000 Nantes, France.

* N. Servagent is with SUBATECH-Ecole des Mines de Nantes, 4, rue Alfred Kastler B.P. 20722 - 44307 Nantes Cedex 3. to flow through the specialized electroreceptive skin. Discrete receptor organs distributed over the epidermis capture an instantaneous 3-dimensional "electric image" of the near environment, comparing the trans-epidermal currents that are expected in the absence of any obstacles with the sensory image that is actually measured. This mode of sensory perception, which is by nature "active", has up to now been very little studied in robotics, despite its natural adaptation for the detection of obstacles in conditions of poor visibility. Based on these potential interests, Mc. Iver and co authors have recently exploited a sensor capable of measuring the difference of potentials between two punctual electrodes, in order to address the problem of electrolocation of small objects through off-line particle filtering [11]. In this case, the sensor's body reduces to a so small surface (two points electrodes between which the difference of potentials is measured) that it does not perturb the electric field produced by another pair of punctual (emitting) electrodes between which, the voltage is imposed. In Angels [1], another technological solution is proposed to the electric sensing. In this case, the sensor is embedded on a realistic 3D body on which each electrode can be polarized with respect to the others through a given voltage $U$. Thus, the sensor sets up an alternating dipole electric field around its body from an emitting electrode on it. This field induces a current in the measurements electrodes. And when, one introduces an object into the media around the sensor, it perturbs the field such that there is a measurable current change in the receiving electrodes. In short, the electric field distortions are then measured through the vector I of the currents flowing across the electrodes and the measurement mode is then qualified of $U-I$. In this terminology, the first letter stands for the emission (here, a voltage $U$ ), the second, for the reception (here a vector of currents I). Thus, in the following we will use the $U-I$ sensor recently proposed in [9] that we distinguish from the $U-U$ sensor of [11]. With this sensor, we will address the problem of the underwater electro-navigation in confined environments, a problem till today unsolved by robotics. For that purpose, we propose a solution based on our sensor's morphology and a sensory-motor loop whose parametrization allows one to achieve conductive objects seeking and insulating obstacles avoidance in a very robust manner without any model as those required by the classical planned approaches [2], [6]. The article is structured as follows. First we will present the sensor technology (section 2 ) as well as the electric test-bed (section 3 ) on which the final experiments will be carried out. Then, we propose in section 4 a solution to our starting navigation problem. The 
solution is then implemented on the experimental test-bed in section 5. Lastly, the article ends with some concluding remarks and perspectives in section 6 .

\section{THE SENSOR}

For more details about the sensor, we refer the reader to [9] and here just remind some basic elements about its physical principle as well as its technology.

\section{A. The sensor principle}

From an external point of view, the sensor is constituted of a body with insulating wetted boundaries on which are fixed a set of $m+1$ macro-electrodes noted $\mathcal{E}_{0}, \mathcal{E}_{1} \ldots \mathcal{E}_{m}$ (Fig. 1). In general each macro-electrode $\mathcal{E}_{\alpha}$ is sub-divided into an array of smaller measurement electrodes denoted $e_{0}, e_{1}, \ldots e_{N}$ except $e_{0}$ which always verifies $e_{0}=\mathcal{E}_{0}$. Thanks to a voltage generator, the potential on $\mathcal{E}_{\alpha=1,2 \ldots m}$ is imposed to be the common ground with respect to which $\mathcal{E}_{0}$ is put under the controlled voltage $U$. Once the sensor immersed in a fluid, this active device produces electric field lines, or equivalently, from $\mathrm{Ohm}$ law, current lines which flow through the fluid surrounding the sensor from $\mathcal{E}_{0}$ to the other macro-electrodes $\mathcal{E}_{\alpha=1, \ldots m}$ (cf. Fig. 1). These current lines are closed through an electric circuit embarked inside the sensor. This circuit measures the vector of the currents $\mathbf{I}=\left(I_{1}, I_{2}, \ldots I_{N}\right)$ flowing across the $e_{i=1,2 \ldots N}$. When an object is placed around the sensor, it generates perturbations of the sensor electric current lines portrait that are measured by this current measurement circuit. The entire device can be embarked on a mobile body as typically an underwater motion controlled vehicle. From the internal point of view, the principle of the $U-I$ sensor is schematized on Fig. 1 , where the squares represent the measurement electrodes. The controlled voltage $U$ is generated by a wave generator $G$ (amplitude $5 \mathrm{~V}$, frequency $22.5 \mathrm{KHz}$ ). The currents flowing across the $e_{i}$ are measured with ammeter-like circuits $A$. Finally, the sensor has a range of about its body length when working in active mode [9]. This range is that observed on fish of the mormyride family.

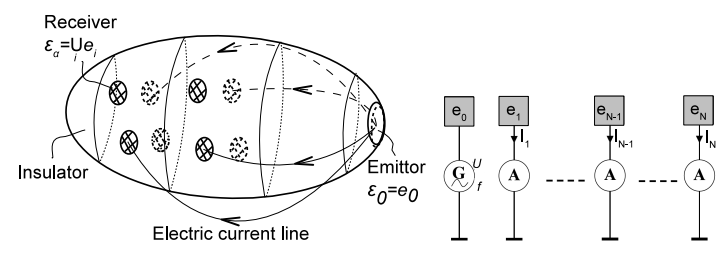

Fig. 1. General principle of the $U-I$ sensor from the external (left) and internal (right) point of view: the macro-electrodes are put under voltage with respect to the emitter. Each receiving macro-electrode is divided into a set of independent measurement electrodes which measure the currents.

\section{B. Sensor morphology}

Based on this principle, we built a first generation of sensors named "slender probes" due to their high aspect ratio (length/thickness) morphology. On these probes, the macroelectrodes $\mathcal{E}_{\alpha}$ are rings or hemispheres which are azimuthally divided into an even number of identical measurement electrodes. As an illustrative example, Fig. 2 shows one of this probes where each of the macro-electrodes (except $\mathcal{E}_{0}$ located in the tail) is divided into a pair of two identical left-right measurement electrodes (it is consequently named the 7electrode probe and such that $\mathcal{E}_{1}=e_{1} \cup e_{2}, \mathcal{E}_{2}=e_{3} \cup e_{4}, \mathcal{E}_{3}=$ $e_{5} \cup e_{6}$, with $\mathcal{E}_{3}$ the head electrode).
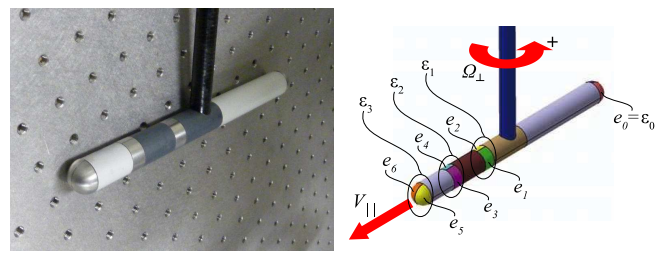

Fig. 2. Picture (left) and schematic view (right) of a 7-electrode senso organized in 4 polarizable rings, 3 of them being divided in two half rings allowing two lateral (left and right) current measurements.

\section{THE ELECTROLOCATION TEST-BED}

\section{A. Tank and cartesian robot}

In order to test our electrolocation sensors and algorithms in controlled and repeatable conditions, an automated test bench consisting in a tank of one cubic meter side with insulating walls and a three-axis cartesian robot has been built (see Fig. 3). The robot fixed on top of the aquarium allows probes positioning in translation along $X$ and $Y$ with a precision of $1 / 10 \mathrm{~mm}$. The orientation in the $(X, Y)$ plane is adjusted in $0.023^{\circ}$ abs using an absolute yaw-rotation stage. All probes tested are positioned in the aquarium at adjustable height using a rigid glass epoxy fibre tube $(\oslash 14 \mathrm{~mm})$. This vertical insulating tube allows the passage of electrical cables dedicated to the signals coming from the electrodes to readout electronics (analogue chain + ADC board) without compromising the measurements. Its verticality is ensured by a micrometric adjustment base. Our test also allows dynamic trajectories management on a significant range of speed relatively to the aquarium size. The maximum speed available is $300 \mathrm{~mm} / \mathrm{s}(\simeq 1 \mathrm{~km} / \mathrm{h})$ for both translations and $80 \% \mathrm{~s}(13.5 \mathrm{tr} / \mathrm{min})$ for rotation. The three axes robot is motion-controlled using simulink software with the dSpace system. The electrolocation signals coming from the electrodes of the probe are, first processed by analogue chain (amplification and filtering) then converted by a 16 bits ADC, $D S 2004$ card, with a resolution of $0.3 \mathrm{mV} / \mathrm{bit}$ (range from $\pm 10 \mathrm{~V})$. This card allows us to convert the 16 channels at the same time with a maximum speed of $1.25 \mathrm{MHz}$.

\section{B. Objects and scenes}

To investigate navigation algorithms in quite complex scenes using electric sense, a set of test objects has been fabricated with conductive and insulating materials. By insulating (or conductive), we mean an ideal material with a conductivity $\gamma$ such that $\gamma / \gamma_{0}=0\left(\right.$ or $\left.\gamma / \gamma_{0}=\infty\right), \gamma_{0}$ 


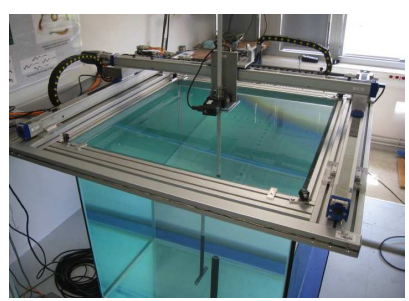

Fig. 3. Electrolocation test bench..

being the conductivity of the ambient fluid (for instance, an ordinary tap water). In practise, plastics (metals) are a good approximation of insulating (conductive) materials. To these simple shaped bodies, we can also add some removable insulating walls. Finally, in the following any scene will be constituted of a combination of these objects and removable walls arbitrarily configured between the four fixed (insulating) walls of the tank.

\section{FROM CLASSICAL TO EMBODIED APPROACH}

Starting from classical approaches where the navigation is planned in a geometric map of the scene around the sensor [2], [6], we have progressively shifted to a more embodied approach where navigation is achieved without any conceptual representation of the world, just through behaviors encoded into simple sensory-motor loops. Such an embodied solution has been achieved in two steps. The first step is based on the exploitation of the sensor morphology, the second, on the observation of living fishes.

\section{A. Exploitation of the sensor morphology}

Even if the embodied navigation does not use any model [8], the modeling of the electric sensor is crucial since it gives the insights helping to guide the seeking of the solution. In particular, in [3] an original modeling approach of the electro-location is proposed. It is based on the method of reflections as it is today developed in the community of diphasic fluid dynamics at low Reynolds numbers [5]. In this approach the electric interactions between the sensor and any object, which are parameterized by the electric potential $\phi$, appear as a super-imposition of successive reflections:

$$
\phi=\phi_{0}+\phi_{1}+\phi_{2}+\ldots,
$$

each $\phi_{i}$ representing a signal traveling from the sensor to the object or conversely (see Fig. 4). As a consequence, each reflection produces a contribution to the total vector of currents, which can be detailed as $\mathbf{I}=\mathbf{I}^{(0)}+\mathbf{I}^{(1)}+\mathbf{I}^{(2)} \ldots$, with $\mathbf{I}^{(i)}=\left(I_{1}^{(i)}, I_{2}^{(i)}, \ldots I_{N}^{(i)}\right)^{T}$ and:

$$
I_{k}^{(i)}=\gamma_{0} \int_{e_{k}} \nabla\left(\phi_{i}\right) \cdot \mathbf{n} d s
$$

where $\mathbf{n}$ is the inward normal to the sensor boundaries and a point denotes a scalar product. Now, due to the rapid attenuation of the reflections with the distance, it is shown in [3] that the vector of total currents can be evaluated with a sufficient precision under the approximation of the second reflection, i.e. with the truncated series expansion:

$$
\mathbf{I} \simeq \mathbf{I}^{(0)}+\mathbf{I}^{(1)}+\mathbf{I}^{(2)},
$$

where $\mathbf{I}^{(0)}$ represents the measured currents with no object in the scene (said vector of "basal currents"), $\mathbf{I}^{(1)}$ is the vector of the currents reflected by the object (with no sensor), i.e. it is a purely geometric component representing the net flux of the field reflected by the objet across the geometric surfaces of the electrodes $e_{k}$. At last, $\mathbf{I}^{(2)}$ represents the electric response that the sensor generates in order to recover its electric equilibrium under the excitation of the first reflection $\phi_{1}$. In [3], we have exploited the symmetry properties of

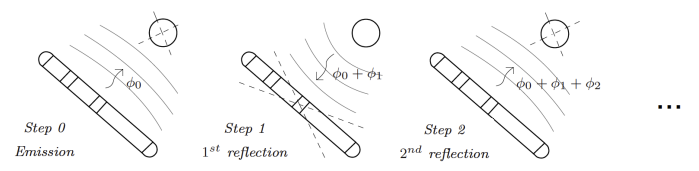

Fig. 4. The principle of the method of the successive reflections. First step (Step 0): we ignore the object (here a small sphere) and we solve the Laplace equation for $\phi_{0}$. Second step (Step 1): this time we ignore the sensor and we solve the Laplace equation for $\phi_{0}+\phi_{1}$ where $\phi_{1}$ is the perturbation of $\phi_{0}$ induced by the object. Third step (Step 2): we solve the Laplace equation for $\phi_{0}+\phi_{1}+\phi_{2}$ ignoring this time the object, $\phi_{2}$ being the perturbation of $\phi_{0}+\phi_{1}$. Each novel potential field can be represented as a new contribution (to $\phi$ ) reflected by the object or the sensor. The method can be pursued but as the interactions fall with the inverse of a positive power of the distance, there is no interest of doing it.

the sensor to write a second decomposition of the total currents as the exclusive summation of a lateral and an axial component:

$$
\mathbf{I} \simeq \mathbf{I}_{a x} \oplus \mathbf{I}_{l a t},
$$

where the axial component (or vector of axial currents) $\mathbf{I}_{a x}$ is axi-symmetric while the lateral component (or vector of the lateral currents) is axi-skew-symmetric. In the first case, the currents measured by any two measurement electrodes of same geometry on a same ring (macro-electrode) are equal. In the second case, the currents flowing across two geometrically identic electrodes azimuthally opposed on a same ring (macro-electrode) are of same strength but of opposite sign (see Fig.2). Thus, it is easy to extract $\mathbf{I}_{a x}$ and $\mathbf{I}_{l a t}$ from the measured currents $\mathbf{I}$, since adding the currents on a same macro-electrode removes the lateral component while subtracting the currents of two azimuthally opposed measurement electrodes removes the axial component. For instance, for the 7 electrode of Fig.2 subtracting the left currents with the right ones give their lateral components while adding them give their axial one. This is essentially due to the fact that the axial and lateral currents are defined as the flux (2) of a radial ${ }^{1}$ and a uniform electric field respectively. Finally, it is shown in [3] that due to the slender morphology of the sensor, we have at the leading order $\mathbf{I}_{a x}=\mathbf{I}_{a x}^{(0)}+\mathbf{I}_{a x}^{(2)}$ and $\mathbf{I}_{l a t}=\mathbf{I}_{l a t}^{(1)}+\mathbf{I}_{l a t}^{(2)}$, with: $\mathbf{I}_{l a t}^{(2)}$ proportional to $\mathbf{I}_{l a t}^{(1)}$, with a

\footnotetext{
${ }^{1}$ i.e. invariant by any rotation around the sensor axis.
} 
coefficient of proportionality strictly positive. But then, from (2) (with " $\sim$ " meaning "proportional to"), we have:

$$
\begin{aligned}
& \mathbf{I}_{l a t} \sim \mathbf{I}_{l a t}^{(1)} \sim \\
& \left(\int_{e_{0}} \nabla \phi_{1} \cdot \mathbf{n}_{\perp} d s, \int_{e_{1}} \nabla \phi_{1} \cdot \mathbf{n}_{\perp} d s, \ldots, \int_{e_{N}} \nabla \phi_{1} \cdot \mathbf{n}_{\perp} d s\right)^{T},
\end{aligned}
$$

with $\mathbf{n}=\mathbf{n}_{\perp}+\mathbf{n}_{\|}$the decomposition of the normal into an axial and a lateral component. Now, since the third member of (5) is nothing but the lateral flux of the electric field reflected by the object, the exploitation of lateral currents for electrolocation is particularly simple. Indeed, the lateral currents obey to a luminous analogy, the surface of the electrodes laterally facing a conducting object is lit by currents flowing in, while the electrodes on the opposite side of the sensor are in the shadow of the outward currents. Conversely, with an insulating object the nearer side of the sensor is shadowed while the opposite side is in the light. As regards the axial perturbative currents, as shown in [3] they are due to the variations of the total resistance of the scene. For instance, if the resistance of the scene globally increases $^{2}$, then the axial currents decrease while if the scene increases its conductivity, the axial currents increase. These properties are summarized on the table I, which indicates how the perturbative currents $\delta \mathbf{I}_{a x}=\mathbf{I}_{a x}-\mathbf{I}_{a x}^{(0)}$ and $\delta \mathbf{I}_{l a t}=$ $\mathbf{I}_{\text {lat }}-\mathbf{I}_{\text {lat }}^{(0)}$ behave in presence of a single object (see [3] for more details). From this table, it is worth noting that $\delta \mathbf{I}_{a x}$ and

\begin{tabular}{|l|c|c|}
\hline$\delta \mathbf{I}_{a x}$ & $>\mathbf{0}$ & for a conducting object \\
\hline$\delta \mathbf{I}_{a x}$ & $<\mathbf{0}$ & for an insulating object \\
\hline$\delta \mathbf{I}_{l a t}$ & $>\mathbf{0}$ & $\begin{array}{c}\text { for a conducting object on the left of the sensor } \\
\text { or } \\
\text { for an insulating object on the right of the sensor }\end{array}$ \\
\hline$\delta \mathbf{I}_{l a t}$ & $<\mathbf{0}$ & $\begin{array}{c}\text { for a conducting object on the right of the sensor } \\
\text { or } \\
\text { for an insulating object on the left of the sensor }\end{array}$ \\
\hline
\end{tabular}

TABLE I

PROPERTIES OF THE PERTURBATIVE CURRENTS $\delta \mathbf{I}_{a x}$ AND $\delta \mathbf{I}_{l a t}$.

$\delta \mathbf{I}_{l a t}$ have a complementary role since the first can be used to determine if the object is conductive or insulating while, once we know the electric nature of the object, the second can allow one to determine if it is on the left or on the right hand side of the sensor. Finally, the axial and lateral components of currents can be easily (in real-time) extracted from the total currents, since by symmetry, subtracting the currents measured by two azimuthally opposed electrodes only retains the lateral currents, while summing all the currents measured by the electrodes of a same ring, kills the lateral currents and only retains the axial ones.

\section{B. Coming back to nature}

The second step of the embodied solution is based on the observation of nature. In fact, it is known from biologists after Hopkins [10] that when an electric fish is placed alone in an empty tank preliminarily equipped with an active

${ }^{2}$ We should add here and after in the same context: "within the range of the sensor". dipole, while the dipole is switched off, the fish peacefully navigates without any apparent objective. On the contrary, as the active dipole is fired so generating an exogenous electric field in the tank, the fish, drastically changes its behavior, now seeking the dipole actively. Furthermore, in order to seek the dipole the fish does not go in straight line but follows the electric lines of the active dipole (see Fig. 5). Although well studied by biologists in the case of the passive sense, this strategy of navigation has only been conjectured in active electro-location [4] where the fields are primarily produced by the fish itself. In this case, the active dipole field of biological experiments should be replaced by the secondary dipoles re-emitted by the objects once polarized by the sensor. Finally, this bio-inspired strategy plays a key role in the embodied solution to the navigation problem.
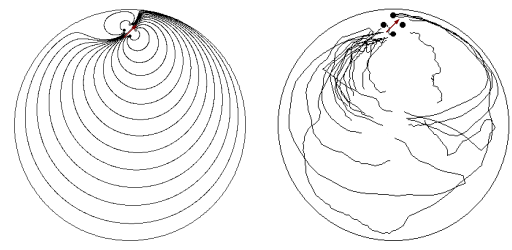

Fig. 5. From Hopkins [10] (left) Electric lines emanating and returning to an active dipole plunged in a tank with circular insulating boundaries. (right) Several fish trajectories recorded by a camera. At each of the trials, the fish seeks the active dipole by following its electric lines.

\section{Sensory-motor loops and behaviors}

In order to extend the previous bio-inspired strategy to the active case, we first of all have to remove from the total currents, the basal component $\mathbf{I}^{(0)}$ and only consider the perturbative component $\delta \mathbf{I}=\mathbf{I}-\mathbf{I}^{(0)}$. As a result, this operation allows the sensor to measure the presence of the objects in its surroundings as if they were active unsteady dipoles $^{3}$. Then, by applying a control law of the general form (3D) :

$$
V_{\|}=\text {cte and: } \boldsymbol{\Omega}_{\perp}=\mathbf{K} \delta \mathbf{I}_{\text {lat }},
$$

with $\mathbf{K}$ a matrix of control gains, one can easily force the sensor to move forward along its axis at the constant scalar velocity $V_{\|}(>0)$, while aligning its axis on the secondary electric lines of $\phi_{1}$ through the lateral rotation of its body $\boldsymbol{\Omega}_{\perp}$. This self alignment is ensured by (5) as $\mathbf{K} \neq \mathbf{0}$ in (6). Now, with the morphology of the 7-electrode sensor of Fig. 2 and all the objects assumed to be in the horizontal equatorial plane of the sensor, we can instantiate the general law (6) with the simpler one:

$$
V_{\|}=\text {cte and: } \Omega_{\perp}=K\left(I_{5}-I_{6}\right),
$$

where $K$, and $\Omega_{\perp}$ are now two scalars, and where the feedback lateral currents reduces to the left minus right difference of the head currents. Now, from the table I, and with our convention on the sign of the currents and

${ }^{3}$ All the objects being at rest, the unsteady character of a dipole is due to the time variations (in a fixed point of space) of the exciting basal field moving with the sensor's body. 
angular velocities, taking $K>0$ in (7) ensures that when a conductive object is on the right (respectively on the left), the sensor turns to the right (respectively to the left). While, when a conductive object is in front of the sensor, the sensor goes forward without changing its orientation ${ }^{4}$. Thus, this law ensures the sensor to be attracted by any conductive object. Now, and still from table 1 , if the object is insulating and on the right (respectively the left), this control law makes it react as if there was a symmetric conductive object on the left (respectively the right). Thus, the feedback law (7) with $K>0$, ensures the sensor to be attracted by the conductive virtual object symmetric of the real insulating one with respect to the sensor axis, i.e. the sensor is repulsed by the insulating object. Finally, this first law achieves the attraction by conductive objects and the repulsion by insulating ones as it is observed in real fish when they are seeking conductive objects as small leaving preys [12]. In the next section, this law is implemented on experiments.

\section{EXPERIMENTAL RESULTS}

In the next subsections, we report some of the experimental results obtained by applying the sensory-motor loop ensuring the behavior: "attracted by conductive objects and repulsed by insulating ones". The experimental conditions are those described in section 3 . The conductivity of water is about $0.041 \mathrm{~S} / \mathrm{m}$, i.e. enough high with respect to plastic and low with respect to metal, to allow considering the objects and walls as ideal insulators or conductors. We progress step by step, starting from the case of the tank with one object only, to the case of complex scenes where several objects are immersed in the tank.

\section{A. Tank with one conductive object}

In this first test, we introduce in the scene a conductive sphere as indicated on Fig. 6(a). From the sensor initial position $A$ to the first one in doted line (point $B$ ), the robot escapes from the insulating corner towards the sphere. The conductive sphere being initially away from the sensor, the detection is first tiny (see Fig. 6(b) where the currents are very weak in $A$ ). Then, from $A$ to $B$, the sensor slightly turns left since it is attracted by the conductive sphere. In $B$, the perturbation induced by the tank's insulating wall becomes predominant compared to that of the conductive object. Consequently, the sensor is repulsed by the wall. In the $C$ sensor's configuration, the sensor avoids a first tank's wall. Being in a corner, the sensor has to avoid a second wall. The configuration denoted by $C$ represents, for this path, the sensor position in which the effect of the corner (i.e. the two walls) is the weakest. From $C$ to $D$, the sensor goes away from the corner and goes towards the most conductive region of the scene: the boundaries of the sphere. When being in the $E$ configuration, the sensor is one length away from the object (from one center to the other), and it detects the conductive region $\left(\delta I_{a x}>0\right)$. From $E$ to

${ }^{4}$ The sensor going forward in a steady scene, only these three cases (object facing the head on left, right or front) are significant since as shown in [3], the lateral currents are negligible in any other case. the final position, the sensor comes closer to the conductive object ( $\delta I_{a x}$ increases) up to the contact.

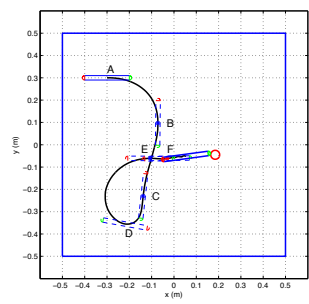

(a) Sensor path in the scene

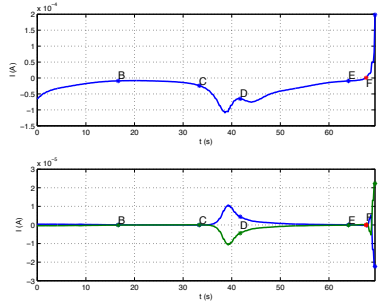

(b) Currents
Fig. 6. Experimental results: Behavior "Attracted by the conductors and repulsed by the insulators" for a tank with a conductive sphere (left) Sensor path for initial position $A$ (right) Time evolution of lateral (top) and axial (bottom) currents.

\section{B. Large insulating object immersed in the tank}

In order to illustrate the effect of large (insulating) objects, we put in the tank a removable wall of $0.38 \mathrm{~m}$ length, in plexiglass material. Its position in the tank is represented in Fig. 7(a). From the initial position $(A)$ to its $B$ position, the sensor seeks the most conductive region within its range. After this point, the robot initiates a cyclic trajectory (Fig. 7(a)) along which the current remains maximum (i.e. the resistance reach a local minimum). In the points $C$ and $D$, the current (Fig. 7(b)) is the weakest of the closed sensor path. Indeed, in the two positions, the sensor goes through in the regions the most confined by the insulating walls. Let us remark that in $C$, the sensor is closer to the removable wall that to the tank's walls. This is due to the fact that in this point, the removable wall being sensed through its edge, it is less influential than in $A$, where the two walls have comparable effects on the measures. Indeed, in this case the sensor is near to be equidistant from the removable and fixed wall.

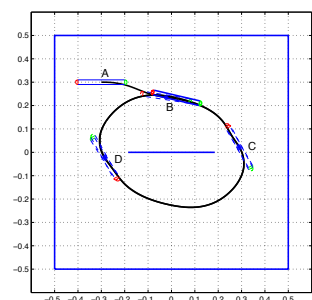

(a) Sensor path in the scene
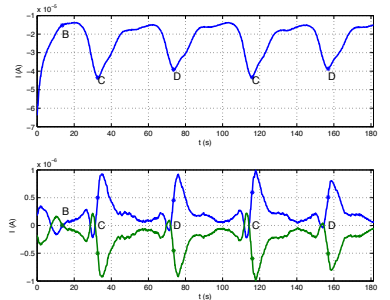

(b) Currents
Fig. 7. Experimental results: Control law "Attracted by the conductors and repulsed by the insulators" for a tank with an insulating wall in its middle (left) Sensor path for initial position $A$ (right) Time evolution of lateral (top) and axial (bottom) currents.

\section{Several (insulating and conducting) objects immersed in the tank}

In its initial position $(A)$, the sensor is immersed in a resistive very confined scene (insulating sphere placed in 
a corner of the tank) (Fig. 8(a)). At point $B$, the sensor comes closer to the insulating sphere and the current strongly decreases (Fig. 8(b)). From $B$ to $C$, the sensor get out of the confined resistive region. From $C$ to $D$, the sensor seeks the most conductive region. From $D$ to $F$, the sensor locks itself in a corner. The point $E$ is the position in which the effect of the corner on the sensor is minimum. At point $F$, the lateral current increases (Fig. 8(b)) since the sensor senses the insulating sphere on its left hand side. From the point $E$ to the point $F$, the sensor seeks the most conductive region of the scene. After the point $F$, the sensor senses the conductive object $\left(\delta I_{a x}>0\right)$ that it finally touches.

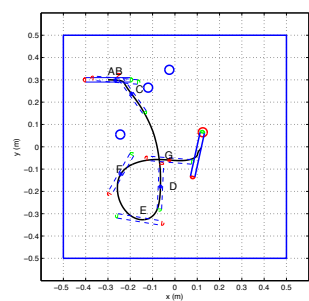

(a) Sensor path in the scene
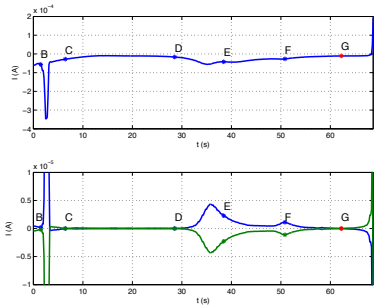

(b) Currents
Fig. 8. Experimental results: Control law "Attracted by the conductor and repulsed by the insulators" for a tank with several objects: 3 insulating spheres and a conductive one (left) Sensor path for initial position $A$ (right) Time evolution of lateral (top) and axial (bottom) currents.

\section{CONCLUSION AND PERSPECTIVES}

In this article we opened new perspectives to solve the problem of underwater navigation in confined environments wetted by turbid waters, where vision and sonar cannot work. The solution is based on a sensor recently proposed in [9]. This sensor is inspired by a natural mode of perception named electric sense. In fish, electric sense is based on the measurement of the distortions by the surrounding of a selfgenerated electric field. These distortions are measured by a distribution of transcutaneous electro-receptors which can be considered as a retina or a skin depending if one insists on the visual, or the haptic modality, of the electric sense. Starting from model-based approaches where the navigation requires the construction of a map of the environment, the Angels project then operated a paradigmatic reorientation based on the concept of embodiment and on the observation of living fishes. From the point of view of embodied intelligence, the navigation problem is investigated as a low level reflex ability of the robot, while pursuing a bio-inspired approach, we implemented a navigation strategy of electric fish which follow the electric lines of active dipoles to seek their preys. In our case, these active fixed dipoles have been successfully replaced by the secondary unsteady dipoles induced by the active polarization of the surrounding by the moving sensor. Thanks to recent progresses in electric modeling and exploiting the morphology of the sensor (in particular its symmetries), it became intuitive to implement a simple sensory-motor loop on currents. This sensory-motor loop allows the robot to seek conductive objects while avoiding the insulating ones. This embodied solution requires no model and exploits the haptic modality of electric sense. From this point of view, electric sense can be considered as touch but with no contact. Indeed, what is interacting here with the surroundings is not the material body (as in haptic) but the electric field generated by the sensor (or the fish) which can be, as such, interpreted as an immaterial extension of the probe's body around it. Today, among the numerous perspectives of these first results, we can mention: the implementation of this behavior on a real autonomous underwater robot, its extension to other behaviors relevant for underwater exploration as "avoiding all objects" or orbiting around them, or again to the multi-agent navigation... In order to address more complex mission, we also study the combination of this embodied navigation with the modelbased approaches.

\section{ACKNOWLEDGMENT}

The ANGELS project is funded by the European Commission, Information Society and Media, Future and Emerging Technologies (FET) contract number: 231845

\section{REFERENCES}

[1] The angels' project, http://www.theangelsproject.eu, 2009

[2] G. Baffet, F. Boyer, and P.B. Gossiaux. Biomimetic localization using the electrolocation sense of the electric fish. In Robotics and Biomimetics ROBIO IEEE, 2008.

[3] F. Boyer, P.B. Gossiaux, B. Jawad, V. Lebastard, and M.Porez. Model for a sensor bio-inspired from electric fish. IEEE transactions on robotics, 2012.

[4] A. Caputi, R. Budelli, K. Grant, and C. Bell. The electric image in weakly electric fish. ii physical images of resistive objects in gnathonemus petersii. Journal of Experimentation in Biologie, 201:pp. 2115-2128, 1998.

[5] J. Happel and H. Brenner. Low Reynolds number hydrodynamics. Prentice Hall, 1965.

[6] V. Lebastard, C. Chevallereau, A. Amrouche, B. Jawad, A. Girin, F. Boyer, and P.B. Gossiaux. Underwater robot navigation around a sphere using electrolocation sense and kalman filter. In IROS 2010 IEEE, 2010.

[7] H.W. Lissmann and K.E. Machin. The mechanism of object location in gymnarchus niloticus and similar fish. The Journal of Experimental Biology, (35):451-486, 1958.

[8] M. Porez, V. Lebastard, A. J. Ijspeert, and F. Boyer. Multy-physics model of an electric fish-like robot: Numerical aspects anbd application to obstacle avoidance. In 2011 IEEE/RSJ Int. Conf. on Intelligent Robots and Systems, (San Francisco, CA), (to appear in), 2011.

[9] N. Servagent, B. Jawad, S. Bouvier, F. Boyer, A. Girin, F. Gomez, V. Lebastard, and P.-B. Gossiaux. Bioinspired sensor for electrolocation and navigation in conducting media. IEEE SENSORS JOURNAL, submitted, 2011

[10] K.T. Shieh, W. Wilson, M. Winslow, D.W. McBride, and C.D. Hopkins. Short-range orientation in electric fish - an experimental study of passive electrolocation. Journal of Experimental Biology, 199:23832393, 1996.

[11] J. Solberg, K. Lynch, and M. MacIver. Active electrolocation for underwater target localization. The International Journal of Robotics Research, 27:529-548, 2008.

[12] G. von der Emde and H. Bleckmann. Finding food : senses involved in foraging for insect larvae in the electric fish gnathonemus petersii. In The Journal of Experimental Biology, volume 201, pages 969-980, 1998.

[13] G. von der Emde, S. Schwarz, L. Gomez, R. Budelli, and K. Grant. Electric fish measure distance in the dark. Letters to Nature, Nature, 395:890-894, 1998. 